Proceedings of the 9 th International Conference on Information Technology and

Applications in Biomedicine, ITAB 2009, Larnaca, Cyprus, 5-7 November 2009

\title{
Question Answering for Biology and Medicine
}

\author{
J. Gobeill, E. Patsche, D. Theodoro, A.-L. Veuthey, C. Lovis and P.Ruch, non-Member, IEEE
}

\begin{abstract}
Biomedical professionals have at their disposal a huge amount of data, such as literature, i.e. textual contents, or databases, i.e. structured contents. But when they have a question, they often have to deal with too many documents in order to efficiently find the appropriate answer in a reasonable time. We have developed a Question Answering system which aims to analyze the user's question, to retrieve the most relevant documents from MEDLINE, and to extract from these retrieved documents a list of candidate answers, ranked by confidence. These candidate answers are concepts issued from biomedical controlled vocabularies, such as the Medical Subject Headings (MeSH) for a first step, and are extracted from the most relevant documents with pattern matching strategies. For evaluation purposes, we apply the system on two biological databases, UniProt and DrugBank. From these resources, we generated two large benchmarks of 200 questions dealing respectively with diseases and proteins, and with diseases and drugs. For these 2 sets, the first candidate answer proposed by our system is respectively correct in $\mathbf{5 7 \%}$ and in $68 \%$, while respectively $70 \%$ and $75 \%$ of all answers to find are contained in the ten first proposed candidate answers. Despite the use of simple Information Extraction strategies, our system exploits the redundancy of information in literature in order to provide a powerful Question Answering system.
\end{abstract}

Index Terms-Question Answering, Information Retrieval, Information Extraction, Ontology

\section{INTRODUCTION}

$\mathrm{Q}$ UESTION-ANSWERING (QA) is the task whereby an automated system returns answers to a question formulated in natural language. QA is a major target since the early ages of Artificial Intelligence [1]. Historically, it often consisted in a knowledge database, in which the answers to a limited set of questions were already encoded; the main challenge was thereof to provide a natural language interface to interact with the database contents. In 1999, the QA Track of the Text REtrieval Conference (TREC) began

Manuscript received June 30, 2009. This work was supported by the Swiss National Science Foundation via the EAGL (Engine for Question Answering in Genomic Literature) and BiND (Biomedical Novelty Detection) grants no. 3252B0-105755 and no. 325200-120758. Knowledge resources for infection diseases have been partially developed thanks to the DebugIT project (grant no. FP7-ICT 217139).

J. Gobeill and Patrick Ruch are with the BiTeM group at the University of Applied Sciences, Information Studies Department, Geneva, Switzerland (corresponding author to provide e-mail: julien.gobeill@ hesge.ch).

E. Patsche and D.Theodoro are with the BiTeM group at the University and Hospitals of Geneva, Geneva, Switzerland.

C. Lovis is with the University and Hospitals of Geneva, Geneva, Switzerland.

A.-L. Veuthey is with the Swiss Institute of Bioinformatics, Geneva, Switzerland. to structure and to rationalize the research on QA from an Information Retrieval perspective [2]. From this perspective, QA systems are supposed to answer any natural language question, by retrieving short text extracts or phrases containing the answer, or the answer itself, out of a large collection of documents. From such a model, QA can be considered as a combination of two basic processes: Information Retrieval and Information Extraction.

As open-domain QA remains a difficult task, many QA systems are developed focusing on a specific domain. Biomedicine is especially designed for this purpose because users in that domain often have at their disposal a huge amount of literature, such as documents stored in the MEDLINE digital library, but often do not have the time, or just the ability, to read all the retrieved documents in order to extract the best answers to their question [3]. Another advantage of the biomedical domain playground for QA systems is the generalized use of rich and highly-structured controlled vocabularies and terminologies; such controlled vocabularies (e.g. the Unified Medical Language System or UMLS) can be used for Information Extraction, in order to extract concepts expressed in a document, and to determine whether a given concept is a possible answer for the user's question or not. For instance, if a user submits a question dealing with diseases, the set of candidate answers can be reduce to diseases concepts within a particular terminological resource.

Many existing biomedical QA systems share fairly similar Information Retrieval engines, as they often rely on MEDLINE in order to retrieve relevant documents. However, the Information Extraction process differs significantly from one system to another. For instance, the QA system described in [4] retrieves relevant documents with PubMed, and then computes a semantic representation of the documents based on formal description logic. Medical entities are then mapped using UMLS, and patterns are used in order to extract candidate answers. The system described in [5] also uses logical forms, but the process is so computation-intensive that it has to be performed offline. These kinds of systems are supposed to extract answers from a document with a very high precision, but are difficult to scale up to large collections in a reasonable processing time.

Another approach is based on redundancy. The success of this approach depends on the idea that the volume of available data is large enough to provide enough quantitative evidence to effectively rank putative answers, found in various contexts [6]. For instance, QA systems described in [7] and [8] retrieve relevant documents with PubMed, in 
order to map correct answers, using specific categorizers. QA systems described in [9] and [10] apply redundancybased methods as well, by mapping possible candidates in the relevant documents, and computing a score using all sources - as a voting process - in order to find the most accurate answers. Unfortunately, if open-domain QA offers some standardized benchmarks thanks to competitions such as TREC, biomedical QA does not yet. Indeed, although QA was on the agenda of the TREC Genomic track, the campaign remained focused on document and passage retrieval. This report is to our knowledge the first study able to provide a sound evaluation of biomedical QA with sizable re-usable benchmarks.

\section{DATA AND METHODS}

\section{A. MEDLINE}

MEDLINE is a digital library in biomedicine, maintained by the United States National Library of Medicine (NLM). It contains about 18 million references of articles from more than 5,000 selected publications, and grows at the rate of 800,000 new references per year. MEDLINE is freely accessible on the Internet and is searchable via PubMed, a search engine provided by the NLM. PubMed is a Boolean search engine that ranks the relevant articles by chronological order. MEDLINE is the daily working tool of biomedical professionals, but the problem of accessing this increasing volume of data creates the need for developing systems that can retrieve and extract highly-relevant contents and not only bibliographical citations.

For each reference, MEDLINE provides usual library meta-data, such as the title, the abstract, the authors, the publication year or the journal together with manually assigned descriptors. These descriptors belong to the Medical Subject Headings (MeSH).

\section{B. UMLS and MeSH}

The MeSH is one of the controlled vocabularies found in the UMLS. The MeSH contains more than 25,000 descriptors. Each descriptor has a preferred form and a unique identifier; for instance, the descriptor Brain Neoplasms has the unique ID D001932. The descriptors are arranged in a hierarchy, and so have one or more tree numbers to allow for classification of concepts in various subtrees; for instance, the tree number C04.588.614.250.195 stands for Brain Neoplasms, which is more precise than C04 (Neoplasms) but less than C04.588.614.250.195.648 (Neurocytoma). In addition, most of descriptors are provided with a list of synonyms or very similar terms, such as Brain Tumors or Cancer of the Brain for Brain Neoplasms. Finally, as being integrated in the UMLS, each descriptor is assigned to one or more semantic types, that are chosen between 135 general categories in the UMLS Semantic Network. For instance, Brain Neoplasm is assigned to only one semantic type, Neoplastic Process (T191), but other semantic types exist for diseases purposes, such as
Anatomical Abnormality (T190) or Mental or Behavioral Dysfunction (T048). Semantic types have their own hierarchic structure, which for example relates T191 to T047 (Diseases) via an is_a relationship.

Whether being collected in the manually assigned descriptors or being automatically extracted from the text, the MeSH provides an efficient way of accessing biomedical information contained in a MEDLINE article [11].

\section{Benchmarks : UniProt and DrugBank}

For evaluating our system, we chose to apply it on two significant benchmarks of 200 questions, generated from two biomedical databases, UniProt and DrugBank.

UniProt is the most comprehensive protein warehouse. It contains more than 5,000,000 protein records. Part of the resource (Swiss-Prot) is manually annotated by professional biocurators, each of them containing biological information about the protein. The UniMed resource is a semiautomatically generated data set, which assigns $\mathrm{MeSH}$ diseases descriptors to proteins involvement in human pathologies [12]. From UniMed, we randomly selected 200 proteins, linked to 245 disease MeSH descriptors. The system had then to propose disease MeSH descriptors in order to answer factoid questions such as "What disease $X$ is caused by protein $Y$ ?". For the protein name, we used the gene name (GN) field of UniProt records.

DrugBank is a richly annotated resource that combines detailed drug data with comprehensive drug target and drug activity description, such as medical indications [13]. From this database, we randomly selected 200 drugs linked to 320 disease MeSH descriptors. The system had to propose disease MeSH descriptors in order to answer questions such as "What disease $X$ is treated by drug $Z$ ?". For the drug name, we used the Brand Name field of DrugBank records.

We thus obtained two separate benchmarks trying to model questions of interest for a wide variety of potential users from clinicians to molecular biologists, biochemists and drug designers.

\section{A three-layer Architecture}

The QA system is based on three components. Given a natural language question, a question categorizer analyzes the question in order to find the question's target set (i.e. the set of MeSH descriptors that are possible answers) and to find the query that will be used for Information Retrieval. Then, given the query, the Information Retrieval component retrieves a set of relevant documents in MEDLINE, using either PubMed or EasyIR, a local search engine in MEDLINE. Finally, given the set of relevant documents and the target set, the Information Extraction component extracts concepts expressed in the most relevant documents, computes a score for each of them, and finally outputs a ranked list of candidate answers.

\section{E. Question categorizer}

The question categorizer uses a Government and Binding 
parser, FIPS [14], which generates a deep syntactic representation of the question.

For a first step, the question categorizer is limited to Whtype questions, such as "What cancers are caused by brcal mutations ?". The question categorizer applies an ordered set of patterns from very specific to more generic ones in order to identify the target set. The most generic pattern selects the noun phrase (NP) found after the wh (e.g. what) and before the auxiliary verb (e.g. are). The NP is parsed to identify its constituents (e.g. head vs adjectives). Then, a category normalizer attempts to associate the longest NP to a list of 450 predefined target types. These target types cover all UMLS semantic types, including aggregation or specification of them. For instance, the noun phrase diseases is associated with 12 UMLS semantic types, for a total of approximately $7500 \mathrm{MeSH}$ descriptors. Very generic descriptors were already manually removed from the list, e.g. Disease for the class Diseases (T047) but better cleaning is needed.

Then, depending on the template question selected by the question categorizers, various keywords (e.g. synonyms of query words) are added to the original user query. The question categorizer also performs stemming, and stopwords recognition and deletion, depending on the search engine selected for the Information Retrieval. Stopwords are particularly needed for PubMed since such Boolean model often returns no document when queries are long.

\section{F. Information Retrieval component}

The goal of the Information Retrieval component is to retrieve a set of relevant documents with regard to the query, from which candidates answer will be computed. The retrieval can be performed via two search engines: PubMed, the NLM search engine accessed via the e-utils, and EasyIR, our local search engine in MEDLINE. EasyIR is based on a locally indexed version of MEDLINE using a linear combination of vector-space search models which showed competitive effectiveness in TREC Genomic tracks [15]. PubMed returns a list of documents ranked by chronological order, while EasyIR returns a list of documents ranked by similarity scores. For online versions of both search engines, the number $K$ of retrieved documents is set to 50 , see explanation in section 3 .

\section{G. Information Extraction component}

The goal of the Information Extraction component is to identify and rank the descriptors belonging to the target set from the ranked set of documents returned by the search engine. The mapping is performed with pattern matching strategies allowing for minor morphological variations (e.g. regular plural forms). We hypothesize that most of the power comes from the redundancy of information and we have not evaluated the impact of more advanced mapping approaches [11]. Thus, we assume that the volume of data in MEDLINE is large enough to supply the correct answers to be found in various retrieved documents under different forms, including those available in the target set. In practice, such a strategy can screen through tens or even hundreds of MEDLINE records in a reasonable time, which is needed for interactive search.

A descriptor score Desc_Score $e_{i}$ is computed for each descriptor $i$ belonging to the target set. For each document $k$, a weight $w_{i, k}$ is computed for the descriptor $i$. $w_{i, k}$ is directly dependent on the frequency of the descriptor in the document $k$ ranging from 0 (the descriptor is not found) to 4 . Descriptors assigned by NLM indexer receive a boosting factor. Then, $w_{i, k}$ is multiplied by the retrieval score $I R \_S c o r e_{k}$ of the document in order to obtain the descriptor score for the document; IR_Score $k_{k}$ is set to 1 when the retrieval was performed via PubMed. For the vector-space model, we use the retrieval status value associated with the document. Finally, the descriptor score of a descriptor $i$ is the sum of the descriptor scores for each document, as showed in (1). Thus, the Information Extraction component returns a ranked list of candidate answers. We expect with this strategy that the correct answers will be mapped in multiple relevant documents, making their score increase, and thus will naturally go up in the ranking.

$$
\text { Desc_Score }_{i}=\sum_{K} w_{i, k} * I R_{-} \text {Score }_{k}
$$

Furthermore, the ranker promotes specific descriptors when ontological information is available. For instance, if Neoplasms and its child Brain Neoplasms are both associated to a document, Neoplasms will be downweighted.

\section{RESUlTS AND DISCUSSION}

The evaluation was conducted with treceval, the program to evaluate TREC results using standards metrics of the competition. Because, we evaluate a QA task and not retrieval task, we selected the following measures: Precision at $0(\mathrm{P} 0)$ and Recall at $10(\mathrm{R} 10)$. $\mathrm{P} 0$ is the precision of the first candidate answer proposed by the system, which is an important feature of QA systems. R10 is the percentage of all correct answers to find proposed by the system in the top ten candidate answers.

\section{A. Use of terminological and ontological resources}

Table 1 shows performances of our system applied on both benchmarks, using either PubMed or the local engine (EasyIR). We establish a first baseline, and we evaluate the following strategies: using MeSH synonyms and using MeSH hierarchy at the Information Extraction level. Best and statistically significant improvements appear in bold. 
TABLE I.

RESULTS OF THE EVALUATION

\begin{tabular}{ccccccccc}
\hline \hline Benchmark & \multicolumn{3}{c}{ UniProt/UniMed } & \multicolumn{3}{c}{ DrugBank } \\
\hline $\begin{array}{c}\text { Search } \\
\text { engine }\end{array}$ & \multicolumn{2}{c}{ PubMed } & \multicolumn{2}{c}{ EasyIR } & \multicolumn{2}{c}{ PubMed } & \multicolumn{2}{c}{ EasyIR } \\
\hline Measure & $\boldsymbol{P O}$ & $\boldsymbol{R} 10$ & $\boldsymbol{P O}$ & $\boldsymbol{R} 10$ & $\boldsymbol{P O}$ & $\boldsymbol{R} 10$ & $\boldsymbol{P O}$ & $\boldsymbol{R} 10$ \\
\hline baseline & 0.46 & 0.65 & 0.46 & 0.62 & 0.57 & 0.68 & 0.63 & 0.73 \\
\hline $\begin{array}{c}\text { exploiting } \\
\text { synonyms }\end{array}$ & 0.49 & 0.64 & 0.48 & 0.63 & 0.57 & 0.74 & $\mathbf{0 . 6 6}$ & $\mathbf{0 . 7 8}$ \\
\hline $\begin{array}{c}\text { exploiting } \\
\text { hierarchy }\end{array}$ & $\mathbf{0 . 5 5}$ & $\mathbf{0 . 6 8}$ & $\mathbf{0 . 5 5}$ & 0.64 & 0.6 & 0.69 & $\mathbf{0 . 6 7}$ & 0.75 \\
\hline \hline
\end{tabular}

Looking at the baseline we observe that results obtained on the DrugBank benchmark outperform those obtained on the UniProt/UniMed resource. Results obtained when using synonyms suggest that such a resource can significantly improve the engine's answering power.

Further, the last row of the table shows that exploiting the MeSH hierarchical structure significantly improves the results obtained on UniMed, although it moderately affects the results on the DrugBank benchmark. It suggests that diseases in DrugBank are defined with more generic categories than in UniMed; for instance, Neurocytoma is never used in the drug indications resource, while there are several occurrences of its ancestor Brain Cancer. Considering that specific concepts are more difficult to extract than more general ones, it can partially explain why results obtained on DrugBank outperformed those obtained on UniMed. Moreover, it means that the MeSH hierarchy is deep enough to express several levels of granularity (e.g. Disease is more general than Neoplasms that is more general than Neurocytoma). Ideally, the optimal strategy shall depend on the granularity required by the application, the task, and ultimately the user's information request.

Finally, we observe that using advanced IR models, which have shown top ranking scores in TREC Genomics competitions, does not always outperform simple Boolean strategies. It remarkably suggests that the redundancy principle seems universal enough to compensate for the likely lost of relevance achieved by the Boolean model. Further analysis of the errors performed by each search model is needed, but a query-dependent selection of the search engine could improve the answer effectiveness of the engine Furthermore, linear combination of answers could be as effective for QA as it is for search engines [15].

\section{B. Effect of corpus size on answering effectiveness}

Table 2 shows the performances of our system when we vary the number of documents selected to compute the answers from a size of 10 to 5000 documents. All experiments were done by exploiting MeSH synonyms and hierarchy. Only results computed on the UniProt/UniMed benchmark are reported since the trend is similar for the benchmark.
TABLE II.

EFFECT OF CORPUS SIZE ON ANSWERING EFFECTIVENESS

\begin{tabular}{ccccc}
\hline \hline Benchmark & \multicolumn{3}{c}{ UniProt benchmark } \\
\hline Search engine & \multicolumn{2}{c}{ PubMed } & \multicolumn{2}{c}{ EasyIR } \\
\hline Measure & PO & R10 & PO & R10 \\
\hline $\mathrm{K}=10$ & 0.49 & 0.63 & 0.47 & 0.58 \\
\hline $\mathrm{K}=20$ & 0.54 & 0.67 & 0.52 & 0.63 \\
\hline $\mathrm{K}=50$ (baseline) & 0.55 & 0.68 & 0.55 & 0.64 \\
\hline $\mathrm{K}=100$ & 0.57 & 0.69 & 0.55 & 0.69 \\
\hline $\mathrm{K}=500$ & $\mathbf{0 . 5 9}$ & 0.71 & 0.56 & $\mathbf{0 . 7}$ \\
\hline $\mathrm{K}=5000$ & $\mathbf{0 . 6 1}$ & $\mathbf{0 . 7 3}$ & $\mathbf{0 . 5 7}$ & $\mathbf{0 . 7 1}$ \\
\hline \hline
\end{tabular}

Again, the results confirm the validity of the redundancy hypothesis. From 50 to 500 the gain can be already statistically significant; up to 1000 , the gain is always significant regarding both recall and precision and whatever engine we select.

\section{CONCLUSION}

We designed and evaluated a new Question-Answering system to help finding answers to natural language questions in medical and biological digital libraries. The evaluation was performed using a benchmark generated from curated legacy databases for molecular biology (UniProt/UniMed) and medicinal chemistry (DrugBank). The system is currently able to find answers with a top precision and recall after ten answers of about $70 \%$ on both benchmarks. These preliminary results confirm that redundancy of information in literature is so high that simple retrieval and answer computation methods can achieve results competitive with more elaborated approaches based on advanced weighting schema inherited from Information Retrieval. In contrast, the use of knowledge-based resources such as terminology and ontology results in a statistically significant improvement.

Current developments include developing query-specific Information Extraction modules such as those needed to detect protein-protein interactions [16] in order to answer more complex questions such as etiological or definitional question. The Question Answering system and most of its components are freely available on the EAGLi platform at $\mathrm{http} / /$ eagl.unige.ch/EAGLi/ (or type "EAGLi" on any web search engine) or upon request to the authors.

\section{REFERENCES}

[1] D. Molla and J. L. Vicedo, "Question Answering in restricted domains: an overview," Computational Linguistics, vol. 33, pp. 4161, 2007.

[2] H. T. Dang, D. Kelly and J. Lin, "Overview of the TREC 2007 Question Answering track," Proceedings of TREC, 2007.

[3] W. R. Hersh et al., "Factors associated with success in searching MEDLINE and applying evidence to answer clinical questions," Journal of the American Medical Informatics Association, vol. 9, pp. 283-293, 2002. 
[4] R. Terol, P. Martinez-Barco and M. Palomar, "a Knowledge based method for the medical question answering problem," Computers in Biology and Medicine, vol. 37, pp. 68-73, 2007.

[5] F. Rinaldi, J. Dowdall, G. Schneider and A. Persidis, "Answering questions in the genomics domain," Proceedings of the ACL 2004 Workshop on Question Answering in Restricted Domains, pp. 46-53, 2004

[6] C. Clarke, G. Cormack and T. Lynam, "Exploiting redundancy in Question Answering," Proceedings of the 24th SIGIR Conference, pp. 358-365, 2001.

[7] D. Demner-Fushman and J. Lin, "answering clinical Questions with knowledge-based and statistical techniques," Computational Linguistics, vol. 33, pp. 63-103, 2007.

[8] Y. Niu, G. Hirst, G. McArthur and P. Rodriguez-Gianolli, "answering clinical Questions with role identification," Proceedings of 41 st Annual Meeting of the Association for Computational Linguistics, Workshop on Natural Language Processing in Biomedicine, pp. 7380, 2003.

[9] A. M. Cohen, J. Yang, S. Fisher, B. Roark and W Hersh, "the OHSU biomedical Question Answering system framework," Proceedings TREC, 2007.

[10] L. Perret, "Question Answering system for French," Lecture Notes in Computer Science, vol. 3491, pp. 392-403, 2005.

[11] D. Triechnigg, P. Pezik, V. Lee, F. de Jong, W. Kraaij and D.RehlozSchuhmann, "MeSH up: effective MeSH text classification for improved document retrieval," Bioinformatics, in press.

[12] A. Mottaz, Y. L. Yip, P. Ruch and A.-L. Veuthey, "Mapping proteins to disease terminologies: from UniProt to $\mathrm{MeSH}$," $\mathrm{BMC}$ Bioinformatics, vol 9, 2008.

[13] D. S. Wishart et al., "DrugBank: a knowledgebase for drugs, drug actions and drug targets," Nucleic Acids Research, vol.36, pp. 901906, 2008.

[14] E. Wehrli, "Fips, a "deep" linguistic multilingual parser," Proceedings of ACL Workshop on Deep Linguistic Processing, pp. 120-127, 2007.

[15] A. R. Aronson et al., "Fusion of knowledge-intensive and statistical approaches for retrieving and statistical approaches for retrieving and annotating textual genomics documents," TREC Genomics Proceedings, 2005

[16] F. Ehrler, "Modular text mining for protein-protein interactions extraction," PhD Thesis, Computer sciences department, University of Geneva, 2009. 